\title{
Electronic Properties of TEM-Sculpted Structure in Graphene
}

\author{
Estelle Kalfon-Cohen, Sagar Bhandari, Robert M. Westervelt and David C. Bell
}

School of Engineering and Applied Sciences, Harvard University, Cambridge, MA 02138

Graphene is an exciting new material composed of single atomic layer of carbon atoms in an hexagonal lattice. Its properties are radically different than traditional semiconductor and they open the way for new science and new applications. Currently, we do not have a precise understanding of how electrons behave along the edges of a graphene sheet. Theoretically, if a current is applied to a sheet of graphene, the majority of electrons that hit the edge of the sheet should scatter back into the sheet because the edge acts as a large potential barrier. Moreover, the atomic configuration along the edge of a graphene sheet can have a large effect on its electronic properties and create new electronic states [1-2]. For instance a triangular structure with zigzag edges is predicted to form magnetic domains [3]. The electron beam in TEM provides an excellent way to sculpt and shaped suspended graphene sheets into atomic scale structures [4].

This approach is also powerful since the same instrument is used to image the structure immediately after it is formed.

The objective of this work is to combine high resolution transmission microscope (TEM) and cooled scanning probe microscope (SPM) to image electron motion and measure the energy of electron states inside atomic-scale graphene. For instance, the edge of a graphene nanoribbon, or thin strip of graphene, has an armchair configuration, its band structure is metallic (no energy gap), but if it takes on a zigzag configuration, the GNR becomes semiconducting (small energy gap). One of the objectives of this project is to determine if SPM, which is a noninvasive technique, can be used instead of TEM to determine the edge configuration by comparison of the empirically determined electronic interactions with theoretical calculations

The approach consists into two main parts. In the first experimental part, the graphene is prepared by mechanical exfoliation (scotch tape technique), and transferred onto a holey Silicon nitride membrane (50 $\mathrm{nm}$ thickness). The suspended sheet in then imaged using Zeiss Libra 200 Cs corrected TEM at $80 \mathrm{KV}$ and structures are sculpted. At $200 \mathrm{kV}$, TEM can be used for high resolution and designing shape in suspended graphene into atomic scale structures. Figure 1 shows a number of shapes that may be sculpted, such as a simple hole, a triangle ( 3 close holes), a square or a thin bridge. Figure 2 shows a TEM image of an atomic scale structure made by nanosculpting a suspended graphene film with a Zeiss TEM instrument. For instance, by making two close holes a ribbon is created with a zigzag atomic configuration.

In the second part, we plan to use our homemade He cooled SPM to measure the conductance at the edge of each atomic structure.

References:

[1] K Nakada, M Fujita, G. Dresselhaus and M. S Dresselhaus, Phy. Rev. B., 54 (1996), 17954.

[2] P Koskinen, S Malola and H Hakknen, Phy. Rev. Lett, 101 (2008), 115502.

[3] WL Wang, SMeng and E Kaxiras, Nano Lett. ,8, (2008), 241-245 .

[4] M Fischenbein and M Drndic, App. Phys. Lett, 93 (2008) 113107. 


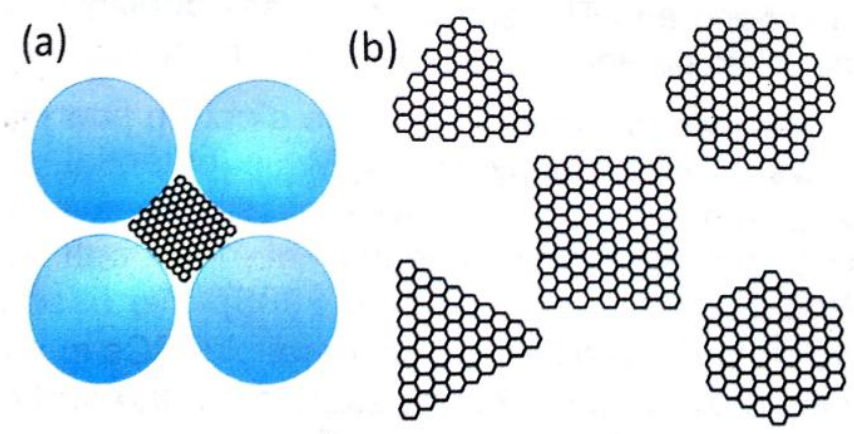

Figure 1. Graphene quantum dot formed by four apertures sculpted by a TEM electron beam (b)Possible atomic configurations of graphene quantum dot with zigzag and armchair atomic edges configuration

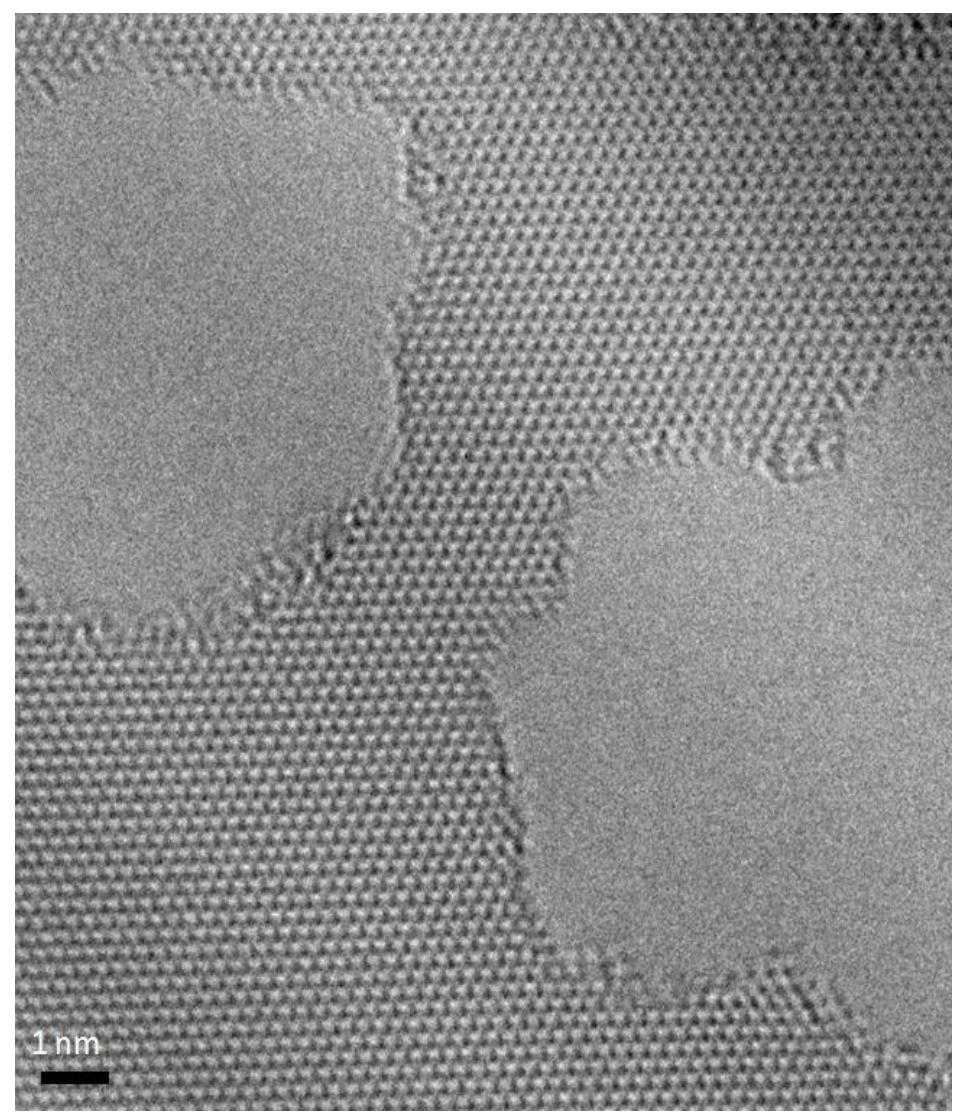

Figure 2. TEM image of an atomic scale structure in a suspended graphene sheet formed by nanosculpting. 